\title{
Accurate and reproducible reconstruction of coronary arteries and endothelial shear stress calculation using 3D OCT: Comparative study to 3D IVUS and 3D QCA
}

\author{
Konstantinos Toutouzas a, 1 , Yiannis S. Chatzizisis b, c, *, 1 , Maria Riga a , \\ Andreas Giannopoulos c, Antonios P. Antoniadis b, c, Shengxian Tu ${ }^{d,}$, , Yusuke Fujino ${ }^{\mathrm{f}}$, \\ Dimitrios Mitsouras ${ }^{g}$, Charalampos Doulaverakis ${ }^{\mathrm{h}}$, Ioannis Tsampoulatidis ${ }^{\mathrm{h}}$, \\ Vassilis G. Koutkias ${ }^{\mathrm{i}, \mathrm{j}}$, Konstantina Bouki ${ }^{\mathrm{k}}$, Yingguang Li ${ }^{\mathrm{d}}$, Ioanna Chouvarda ${ }^{\mathrm{i}, \mathrm{j}}$, \\ Grigorios Cheimariotis i, j, Nicos Maglaveras i, j, Ioannis Kompatsiaris ${ }^{\text {h }}$, Sunao Nakamura ${ }^{f}$, \\ Johan H.C. Reiber ${ }^{\mathrm{d}}$, Frank Rybicki ${ }^{\mathrm{g}}$, Haralambos Karvounis ${ }^{\mathrm{c}}$, Christodoulos Stefanadis ${ }^{\mathrm{a}}$, \\ Dimitris Tousoulis ${ }^{\mathrm{a}}$, George D. Giannoglou ${ }^{\mathrm{C}}$
}

${ }^{a}$ First Department of Cardiology, Hippokration Hospital, Athens University Medical School, Athens, Greece

b Cardiovascular Division, Brigham and Women's Hospital, Harvard Medical School, Boston, MA, USA

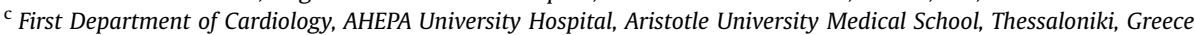

d Division of Image Processing, Department of Radiology, Leiden University Medical Center, Leiden, The Netherlands

e Biomedical Instrument Institute, School of Biomedical Engineering, Shanghai Jiao Tong University, Shanghai, China

${ }^{\mathrm{f}}$ Department of Cardiology, New Tokyo Hospital, Chiba, Japan

${ }^{g}$ Applied Imaging Science Laboratory, Department of Radiology, Brigham and Women's Hospital, Harvard Medical School, Boston, MA, USA

h Information Technologies Institute, Centre for Research and Technology Hellas, Thessaloniki, Greece

${ }^{i}$ Laboratory of Medical Informatics, Aristotle University Medical School, Thessaloniki, Greece

${ }^{\mathrm{j}}$ Institute of Applied Biosciences, Centre for Research and Technology Hellas, Thessaloniki, Greece

${ }^{\mathrm{k}}$ Second Department of Cardiology, General Hospital of Nikaia, Piraeus, Greece

\section{A R T I C L E I N F O}

\section{Article history:}

Received 29 January 2015

Received in revised form

15 March 2015

Accepted 6 April 2015

Available online 13 April 2015

\section{Keywords:}

Optical Coherence Tomography

Intravascular Ultrasound

Quantitative Coronary Angiography

Shear Stress

Method comparison study

\begin{abstract}
A B S T R A C T
Background: Geometrically-correct 3D OCT is a new imaging modality with the potential to investigate the association of local hemodynamic microenvironment with OCT-derived high-risk features. We aimed to describe the methodology of 3D OCT and investigate the accuracy, inter- and intra-observer agreement of $3 \mathrm{D}$ OCT in reconstructing coronary arteries and calculating ESS, using 3D IVUS and 3D QCA as references.

Methods-Results: 35 coronary artery segments derived from 30 patients were reconstructed in 3D space using 3D OCT. 3D OCT was validated against 3D IVUS and 3D QCA. The agreement in artery reconstruction among 3D OCT, 3D IVUS and 3D QCA was assessed in 3-mm-long subsegments using lumen morphometry and ESS parameters. The inter- and intra-observer agreement of 3D OCT, 3D IVUS and 3D QCA were assessed in a representative sample of 61 subsegments ( $\mathrm{n}=5$ arteries). The data processing times for each reconstruction methodology were also calculated. There was a very high agreement between 3D OCT vs. 3D IVUS and 3D OCT vs. 3D QCA in terms of total reconstructed artery length and volume, as well as in terms of segmental morphometric and ESS metrics with mean differences close to zero and narrow limits of agreement (Bland-Altman analysis). 3D OCT exhibited excellent inter- and intra-observer agreement. The analysis time with 3D OCT was significantly lower compared to 3D IVUS. Conclusions: Geometrically-correct 3D OCT is a feasible, accurate and reproducible 3D reconstruction technique that can perform reliable ESS calculations in coronary arteries.
\end{abstract}

(c) 2015 Elsevier Ireland Ltd. All rights reserved.

\footnotetext{
* Corresponding author. Cardiovascular Division, Brigham and Women's Hospital,

Harvard Medical School, 75 Francis Street, Boston, MA, USA.

E-mail address: ychatzizisis@icloud.com (Y.S. Chatzizisis).

1 The first two authors contributed equally to the manuscript.
} 


\section{Introduction}

Coronary artery imaging is fundamental for the diagnosis and treatment of coronary artery disease. IVUS (intravascular ultrasound) is a robust invasive imaging technique that allows accurate visualization of arterial lumen and atherosclerotic plaque and has substantially expanded our insights into the pathophysiology of atherosclerosis, vascular remodeling and in-stent restenosis [1]. IVUS has comparatively low in-plane spatial resolution $(100-150 \mu \mathrm{m})$ and therefore provides limited information about fibrous cap thickness and inflammation. Intravascular OCT (optical coherence tomography) is a newer invasive imaging modality that offers 10-fold superior in-plane resolution (10-15 $\mu \mathrm{m})$ than IVUS, enabling a more accurate evaluation of the cross-sectional microstructure of coronary artery wall and stents [2].

Local coronary hemodynamics, including ESS (endothelial shear stress), are causally related to the localization and progression of atherosclerosis $[3,4]$. A detailed assessment of blood flow properties in coronary arteries requires accurate $3 \mathrm{D}$ imaging of the arterial lumen. Geometrically-correct 3D IVUS based on the fusion of IVUS with biplane angiography has been used for more than a decade for this purpose. This technique has been proven feasible, accurate and reproducible and has advanced our knowledge on the role of ESS in vascular remodeling and natural history of atherosclerosis [5-9]. 3D QCA (Quantitative Coronary Angiography) is another wellvalidated imaging technique that confers $3 \mathrm{D}$ reconstruction of coronary arteries utilizing two angiographic planes [10]. Even though 3D IVUS and 3D QCA can accurately calculate the local hemodynamic microenvironment [5,11] they provide limited information about the pathobiologic nature of plaque. Combined evaluation of local hemodynamic factors [i.e. ESS, FFR (fractional flow reserve)] with plaque morphologic features could potentially improve our ability to identify high-risk plaque creating new diagnostic and therapeutic perspectives for coronary artery disease $[12,13]$. Geometrically-correct 3D OCT based on the fusion of biplane angiography with OCT may enable the investigation of the association of local hemodynamic microenvironment with OCTderived high-risk features, such as thin fibrous cap, inflammation and lipid pool, providing incremental diagnostic and prognostic information.

Despite sporadic reports on the feasibility of geometricallycorrect 3D OCT reconstruction [14-16], the accuracy and reproducibility of 3D OCT vs. 3D IVUS and 3D QCA have not been systematically investigated. In this study we sought to: (i) describe the methodology of geometrically-correct 3D OCT, (ii) investigate the accuracy of 3D OCT in reconstructing coronary arteries and calculating ESS, using 3D IVUS and 3D QCA as reference, and (iii) investigate the inter- and intra-observer agreement of 3D OCT.

\section{Methods}

\subsection{Study population}

The characteristics of the study population are outlined in Supplemental Table 1 . We studied 35 coronary arteries [LAD (left anterior descending artery), $\mathrm{n}=23$; LCX (left circumflex artery), $\mathrm{n}=5$; RCA (right coronary artery), $\mathrm{n}=7$ ] derived from 30 patients who underwent a clinically indicated cardiac catheterization as described in Supplemental Table 1. The study included both native (primarily) and stented coronary artery segments. Imaging data were acquired in Hippokration Hospital, Athens, Greece, in General Hospital of Nikaia, Piraeus, Greece and in New Tokyo Hospital, Chiba, Japan using standardized image acquisition protocols. Coronary imaging preceded percutaneous interventions. Data analysis was blinded to the data acquisition process. The local Institutional
Ethics Committees approved the study. All study subjects provided written informed consent for their participation in the study.

\subsection{Study design}

According to the study design (Fig. 1), all coronary arteries $(\mathrm{n}=35)$ underwent OCT imaging and geometrically-correct 3D OCT reconstruction as described below. In 10 of those arteries additional IVUS imaging was performed prior to OCT. Those arteries were reconstructed with geometrically-correct 3D IVUS as detailed below and served as validation cohort of 3D OCT vs. 3D IVUS. Furthermore, 7 of the validation cohort arteries that were imaged with OCT and IVUS underwent 3D QCA analysis according to the protocol below. Thus the final study included $n=10$ arteries (LAD, $\mathrm{n}=7$; LCX, $\mathrm{n}=2$; RCA, $\mathrm{n}=1$ ) for the validation of 3D OCT vs. 3D IVUS and $\mathrm{n}=7$ arteries (LAD, $\mathrm{n}=4$; $\mathrm{LCX}, \mathrm{n}=2$; RCA, $\mathrm{n}=1$ ) for the validation of 3D OCT vs. 3D QCA. The agreement of 3D OCT vs. 3D IVUS, 3D QCA and 2D angiography, as well as the inter- and intraobserver agreement of each 3D reconstruction modality were evaluated using morphologic and local hemodynamic metrics. Detailed quality assessment of imaging data was initially performed to minimize the effect of severe image artifacts on our results. No data were excluded based on the quality assessment.

\section{3. $3 D O C T$} Fig. 1:

The principal steps of the 3D OCT methodology are depicted in

\subsubsection{Raw data acquisition and pre-processing}

The OCT acquisition was performed with a frequency domain OCT imaging system (C7-XRT OCT Intravascular Imaging System, Westford, MA, USA) at a pullback speed of $20 \mathrm{~mm} / \mathrm{s}$, axial resolution of $15 \mu \mathrm{m}$ and frame rate of 100 frames/sec. Temporary blood clearance was achieved with contrast infusion. The refractive index was automatically corrected by the OCT imaging system [2]. The OCT catheter diameter was also automatically corrected by the machine and manually corrected by the OCT operator as needed. Before the start of the OCT pullback a coronary angiogram was obtained in two perpendicular angiographic projections, i.e. RAO (Right Anterior Oblique) $30^{\circ}$ and LAO (Left Anterior Oblique) $60^{\circ}$ with $<5^{\circ}$ caudal or cranial angulation (Hicor ACOM TOP, Siemens Medical Solutions AG, Forchheim, Germany; Integris Allura 9 and Allura Clarity FD, Philips Medical Systems BV, Eindhoven, the Netherlands). From each angiographic projection, a single enddiastolic frame was manually selected using the simultaneous ECG recordings. For the conversion of pixel size to $\mathrm{mm}$, a scaling factor was determined using the $6 \mathrm{~F}$ guide catheter as reference.

\subsubsection{OCT images segmentation}

A sampling rate of 1 to 2 or 1 to 3 OCT images was applied to account for ECG gating issues. OCT images were calibrated using the digital image pixel size. The lumen was semi-automatically detected on the OCT images using the core functionality of IVUSAngio Tool, a publicly available software for endovascular image processing (IVUSAngioTool 2.1, Thessaloniki, Greece, http://mklab. iti.gr/ivus) [17].

\subsubsection{Lumen $3 D$ reconstruction}

This step was implemented in Rhinoceros ${ }^{\circledR}$, version 5.0 evaluation, Robert McNeel \& Associates, Seattle, Washington, USA. The lumen borders were semi-automatically detected in each angiographic projection and the lumen centerline was calculated in each projection. The 3D centerline of the artery was extracted from the two angiographic projections and the segmented OCT frames were 


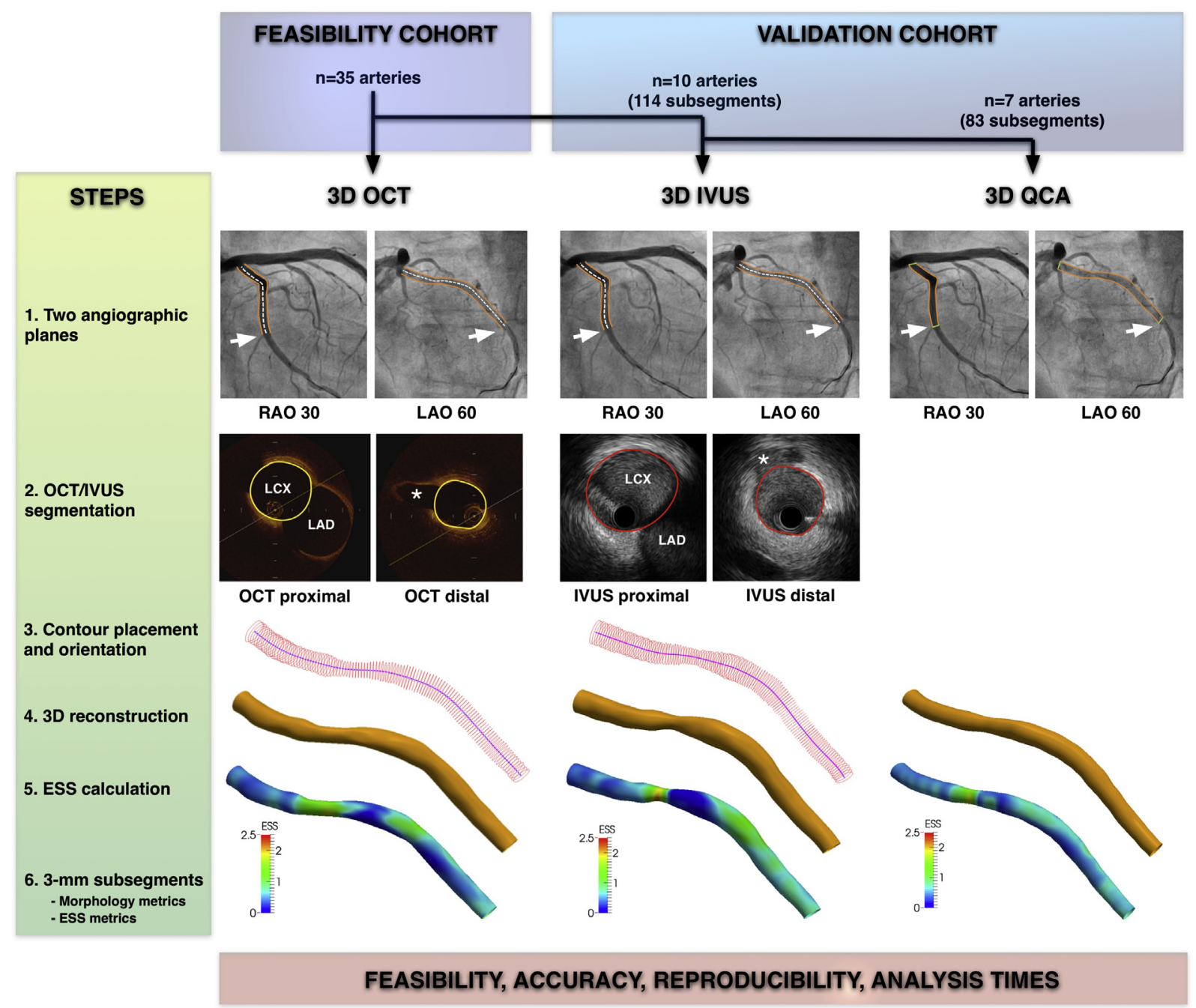

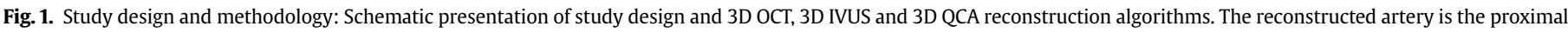
segment of LCX from the left main bifurcation to the take-off of the atrioventricular groove LCX (white arrows).

placed perpendicularly with the lumen centroid of each frame intersecting the 3D centerline $[14,18]$. The relative rotation of each OCT frame with respect to the 3D centerline was assessed using the sequential triangulation method [19]. The absolute rotation of the entire set of OCT contours relative to the 3D centerline was determined by successive rotations and back-projections on each angiographic plane [6]. The angle that presented the smallest cumulative error for both back-projections was the absolute rotation angle of the contours. Lumen contours were connected by spline curves and a non-uniform rational B-spline surface was utilized to rebuild the lumen surface in space. The resulting 3D lumen volume was exported to the CFD (computational fluid dynamics) solver.

\section{4. $3 D$ IVUS}

The geometrically-correct 3D IVUS was performed using the IVUSAngio tool (IVUSAngioTool 2.1, Thessaloniki, Greece, http:// mklab.iti.gr/ivus) as previously described and validated (Fig. 1) [17]. To ensure the validity of comparison between 3D OCT and 3D IVUS, all steps of 3D IVUS were identical to 3D OCT, except that instead of segmenting OCT images we segmented IVUS images. Briefly, the IVUS acquisition was performed with a mechanical imaging system (iLab, Boston Scientific, Natick, Massachusetts, USA) at a pullback speed of $0.5 \mathrm{~mm} / \mathrm{s}$ and a maximum frame rate of
30 frames/sec. The IVUS catheter (Atlantis SR Pro and OptiCross, Boston Scientific, Natick, MA, USA) was inserted through a 6 F guide catheter into the studied coronary artery and with the IVUS catheter at the most distal position, two perpendicular angiographic projections were acquired using exactly the same angulations as with 3D OCT. Angiographic image calibration was applied using the $6 \mathrm{~F}$ guide catheter as reference. A single end-diastolic frame was manually selected in each angiographic projection and the lumen borders were semi-automatically outlined. The lumen centerline was identified in each projection and the 3D centerline of the artery was reconstructed using the $2 \mathrm{D}$ tracings. IVUS images were calibrated using the digital image pixel size. The lumen borders were identified on end-diastolic IVUS frames and the segmented IVUS contours were placed perpendicularly along the 3D reconstructed centerline. Relative and absolute rotation of IVUS contours were performed and the geometrically-correct 3D lumen surface was built, using spline curves and mesh grid. The 3D lumen mesh was finally exported to the CFD solver.

\section{5. $3 D Q C A$}

The 3D angiographic reconstruction was performed using a commercially available 3D QCA software package (QAngio XA 3D RE, Medis Specials bv, Leiden, the Netherlands; Fig. 1) [10]. 
Automatic calibration was applied using the digital image pixel size. For the artery reconstruction we used the same angiographic projections used for the 3D OCT and 3D IVUS. One to three anatomical markers (e.g. bifurcations) were identified as reference points in the two angiographic projections for the automated correction of angiographic system distortions. Automated 2D lumen edge detection was performed and the lumen surface was reconstructed in 3D after refining the correspondence between the two projections. The resulting 3D lumen mesh was exported to the CFD solver.

\subsection{Matching of $3 D$ OCT, $3 D$ IVUS and $3 D Q C A$}

To account for the effect of coronary motion on longitudinal displacement of OCT and IVUS catheters making the 3D OCT, 3D IVUS and 3D QCA reconstructions comparable, we meticulously identified identical start, middle and end landmark points on the angiograms, OCT and IVUS pullbacks [20]. For most patients the proximal landmarks were the RCA ostium and left main bifurcation, the middle landmarks were side-branches and the distal landmark were carefully matched side branches or radiopaque points along the OCT and IVUS catheter (Fig. 1).

\subsection{Accuracy of 3D OCT vs. 2D angiography}

To assess the accuracy of 3D OCT we back-projected the reconstructed lumen on each angiographic projection and calculated the agreement of the back-projected lumen with the actual angiographic lumen using the latter as reference (Fig. 2). The point where each OCT frame intersected the lumen 3D centerline was set as the control point [21]. The entire set of contours was rotated consecutively by two-degree steps until completion of a full circle (i.e. 180 rotations). In each rotation the reconstructed lumen was backprojected on the RAO $30^{\circ}$ and LAO $60^{\circ}$ plane and the diameters of the back-projected lumen and angiographic lumen were calculated at each control point. The agreement between the diameters was used as metric of the accuracy of 3D OCT vs. 2D angiography.

\subsection{Accuracy of 3D OCT vs. 3D IVUS and $3 D$ OCT vs. 3D QCA: morphology metrics}

The agreement in artery reconstruction among the three imaging modalities was assessed using morphology and ESS metrics. The morphology metrics included total lumen length and volume, as well as segmental variables calculated in $3 \mathrm{~mm}$-long subsegments across the reconstructed artery. In each subsegment the average lumen area, lumen volume, minimum diameter and maximum diameter were calculated as metrics of artery size, whereas the centerline curvature was calculated as metric of lumen shape. For the calculation of those morphology metrics each 3-mmlong subsegment of the reconstructed artery was sectioned in six 0.5 -mm-long intervals, yielding six cross-sections per subsegment. The lumen area of each $3-\mathrm{mm}$ subsegment was calculated as the average lumen area of the six $0.5-\mathrm{mm}$ cross-sections contained in the subsegment. In each of these cross-sections, the diameter was calculated in 9-degree increments along the luminal circumference and the maximum and minimum diameters were identified. The centerline curvature was calculated in $0.5-\mathrm{mm}$ intervals as the reciprocal of the radius of the osculating circle at each centerline location and then averaged over each 3-mm subsegment.

\subsection{Accuracy of $3 D$ OCT vs. 3D IVUS and $3 D$ OCT vs. 3D QCA: ESS metrics}

Further to morphologic metrics, the agreement across the 3D
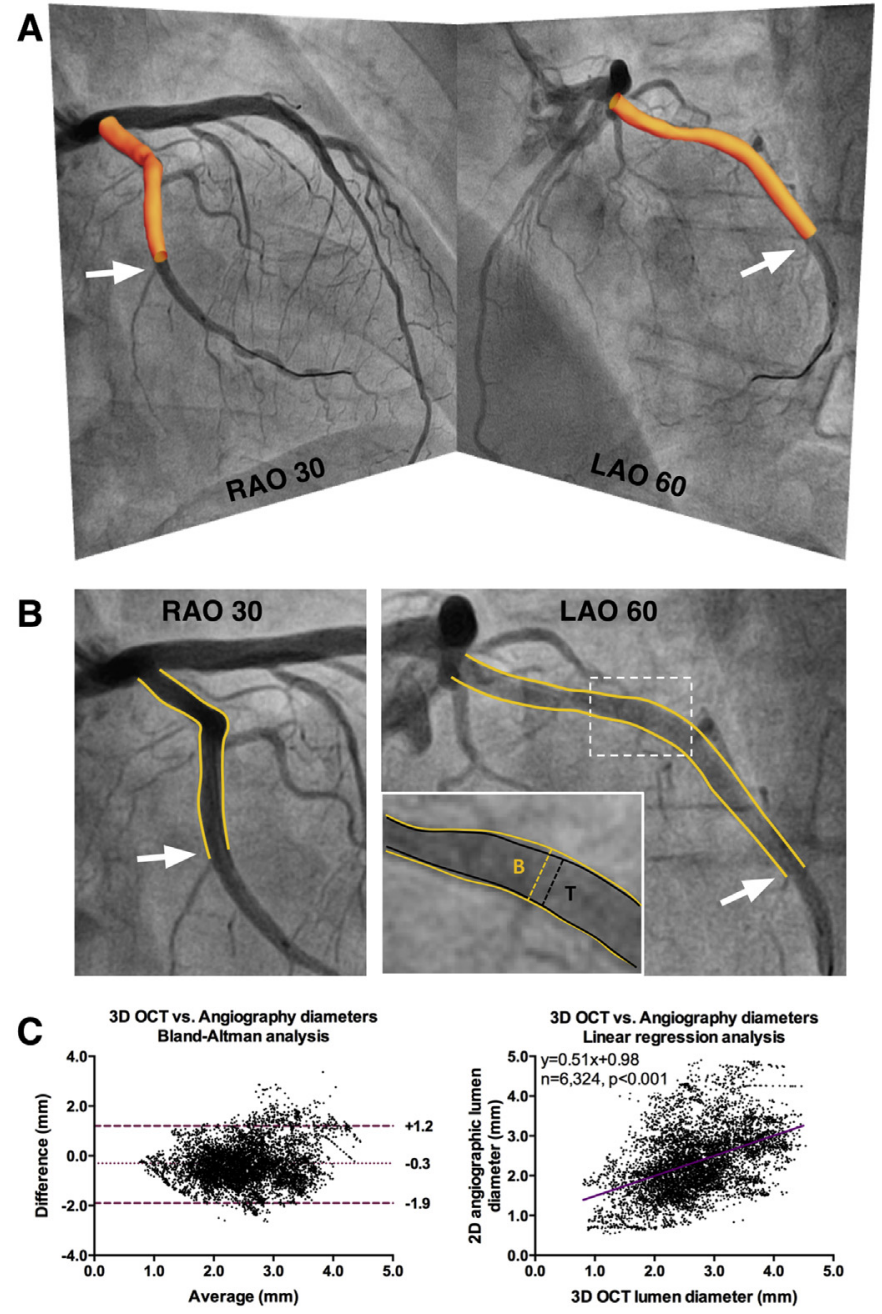

Fig. 2. $3 \mathrm{D}$ OCT back-projection on two orthogonal angiographic planes: A. Same segment with Fig. 1 (LCX) showing the agreement of 3D OCT back-projections on RAO $30^{\circ}$ and LAO $60^{\circ}$ with actual angiographic lumen. The LCX was reconstructed from the left main bifurcation to the origin of atrioventricular groove LCX (white arrows), B, Back-projected lumen borders on RAO $30^{\circ}$ and $\mathrm{LAO} 60^{\circ}$. The insert provides an example of back-projected (orange dashed line, B) and actual (black dashed line, T) lumen diameters, $C$. Bland-Altman and linear regression plots showing quantitatively the agreement between geometrically-correct 3D OCT and 2D angiography (For interpretation of the references to color in this figure legend, the reader is referred to the web version of this article.)

reconstructions was assessed using ESS metrics. The ESS was calculated in the reconstructed arteries using CFD as previously described $[8,22,23]$. In particular, the governing Navier-Stokes flow equations for the conservation of mass and momentum were solved with a finite volume numerical code (Fluent 14.5, ANSYS Inc., PA, USA). All CFD analyses were performed in a dedicated workstation operating in Microsoft Windows 8 environment with Intel i7 quad core $2 \mathrm{GHz}$ processor and $8 \mathrm{~GB}$ RAM. The lumen volume was populated with a dense mesh of tetrahedral/hexahedral elements plus five prismatic layers at the lumen boundary to capture near-wall effects. Parabolic flow profile was implemented at the flow inlet. Coronary blood flow and inlet velocity were calculated for each separate case. Coronary blood flow was estimated from the number of angiographic frames required for contrast material to fill the $3 \mathrm{D}$ reconstructed lumen volume [8]. The inlet velocity was calculated as the ratio of blood flow over the inlet surface area. The nature of the flow was assumed as 3D, steady, laminar, isothermal with no external forces applied on it and the arterial wall was considered as non-elastic and impermeable. The blood was 
considered as non-Newtonian fluid obeying to the power law and the blood density was considered to be constant.

The ESS and lumen radius raw data were presented in 2D maps with the artery length $(\mathrm{mm})$ in $\mathrm{x}$ axis and the circumference (degrees) in $\mathrm{y}$ axis after converting the Cartesian coordinates to curvilinear (Fig. 3). The artery was divided into 3-mm-long subsegments and in each subsegment we calculated the total mean ESS of the entire segment, as well as the lowest and highest mean ESS values in 30-degree-arcs across the lumen circumference [23].

\subsection{Inter- and intra-observer agreement of $3 D$ OCT, 3D IVUS and $3 D Q C A$}

The inter-observer agreement of 3D OCT, 3D IVUS and 3D QCA was assessed by two independent experts in a representative sample of 5 randomly selected arteries. The intra-observer agreement of 3D OCT, 3D IVUS and 3D QCA was assessed in the same sample of 5 arteries by the first expert at two time points one month apart to minimize the recall bias. Of note, the experts that performed the QCA analysis were different than the experts who analyzed the OCT and IVUS images. The artery size, shape and ESS variables as described above were used as metrics of inter- and intra-observer agreement.

\subsection{Data processing times}

The data processing times from raw data acquisition to 3D reconstruction and CFD were compared across the three reconstruction modalities. To ensure the validity of the comparisons identical software, hardware and standardized protocols were used for data acquisition, image segmentation and CFD for each case.

\subsection{Statistical analysis}

Statistical analysis was performed with the statistical package
GraphPad Prism 6.0 (GraphPad Inc., San Diego, CA, USA) and IBM SPSS Statistics 21.0 (IBM Corp., New York, NY, USA). All results were expressed as mean \pm standard error of mean for continuous variables and absolute number (\%). The method comparison study among 3D OCT, 3D IVUS and 3D QCA, as well as the inter- and intraobserver agreement of each modality were assessed with Bland-Altman analysis [24], coefficient of determination $\left(r^{2}\right)$ and mixed effects linear regression analysis using patient and artery as random effects. In Bland-Altman plots, the absolute differences between corresponding measurements (y axis) were plotted against their mean ( $x$ axis). The mean difference (bias) and the limits of agreement (mean $\pm 1.96 \mathrm{SD}$ ) were calculated. The 3D OCT was considered to agree with the 3D IVUS and 3D QCA when the mean differences and limits of agreement of 3D OCT vs. 3D IVUS and 3D OCT vs. 3D QCA were comparable to the inter- and intraobserver agreement of 3D IVUS and 3D QCA, respectively. Sensitivity, specificity, positive and negative predictive values were calculated to assess the agreement of ESS measurements across the reconstruction methods. The cut-off points for low and higher ESS were set to the 50th percentile of the frequency distribution. The comparison of analysis times among methods was done with repeated-measures ANOVA using Tukey test for multiple comparisons. $\mathrm{P} \leq 0.05$ was considered as the level of significance.

\section{Results}

3D OCT was successfully implemented in all study arteries $(\mathrm{n}=35$; mean length $=37.8 \pm 1.7 \mathrm{~mm}$, range from 17.5 to $53.4 \mathrm{~mm}$ ). The length of the reconstructed arteries was very similar to the length of OCT pullbacks (bias $0.2 \mathrm{~mm}$, limits of agreement $-3.8-3.4 \mathrm{~mm}, \mathrm{y}=1.01 \mathrm{x}-0.13, \mathrm{p}<0.001)$. Similarly, 3D IVUS and 3D QCA were successfully implemented in 10 and 7 arteries, respectively. The segmental analysis was done in $1143-\mathrm{mm}-$ long subsegments for the 3D OCT vs. 3D IVUS validation and in 83 3-mm-long subsegments for the 3D OCT vs. 3D QCA validation.
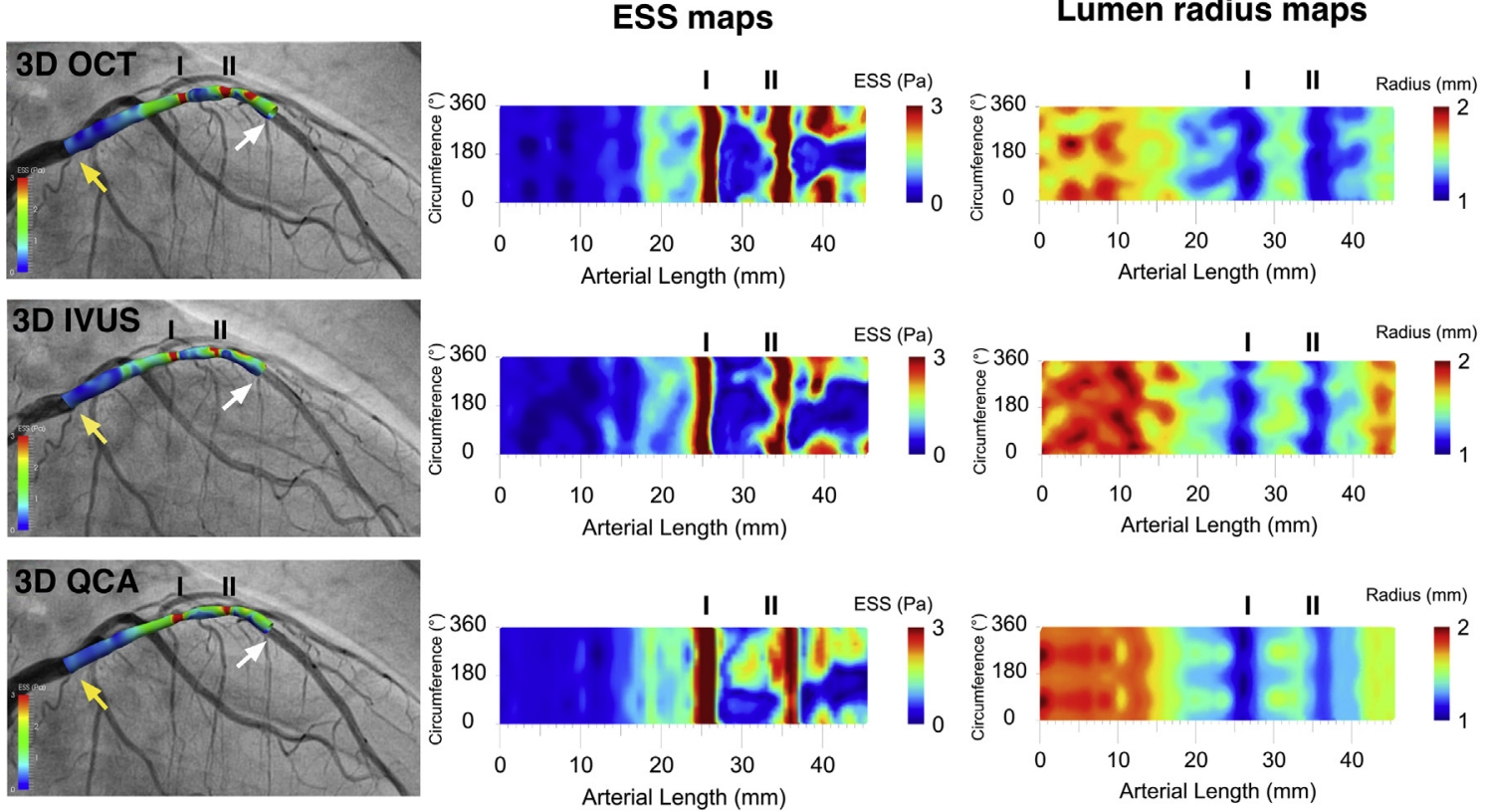

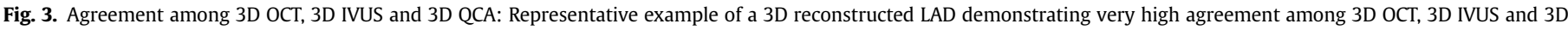

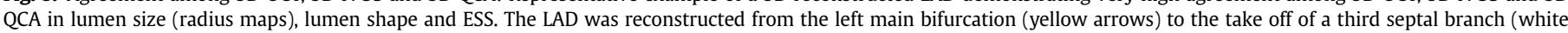

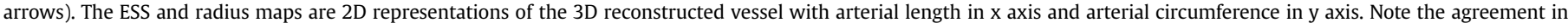

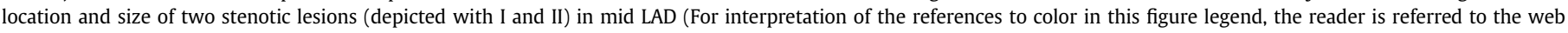
version of this article.) 


\subsection{Accuracy of $3 D$ OCT vs. $2 D$ angiography}

The 3D reconstructed coronary arteries $(n=35)$ from the fusion of OCT and angiography were back-projected on the 2D angiographic planes as shown on Fig. 2 and the diameters of the backprojected lumen and angiographic lumen were compared with each other. There was good agreement between the back-projected and angiographic lumen diameters (mean difference $-0.3 \mathrm{~mm}$, limits of agreement from $-1.9 \mathrm{~mm}$ to $1.2 \mathrm{~mm}, \mathrm{y}=0.51 \mathrm{x}+0.98$, $\mathrm{n}=6324$ control points, $\mathrm{p}<0.001$; Fig. 2 C).

\subsection{Accuracy of 3D OCT vs. 3D IVUS: morphology metrics}

Geometrically-correct 3D OCT was compared with geometrically-correct 3D IVUS in the validation cohort $(n=10)$, using the latter as reference (Fig. 3). There was a significant agreement between 3D OCT and 3D IVUS in terms of total reconstructed artery length and volume (Table 1). In the segmental analysis $(\mathrm{n}=114)$ the 3D OCT yielded similar lumen areas, volumes and diameters compared to 3D IVUS with mean differences between methods close to zero, narrow limits of agreement, very high $r^{2}>0.80$ (with the exception of curvature) and slopes close to one and intercepts close to zero in linear regression analysis (Table 1 ). In the same fashion, the 3D shape of the OCT-based reconstructions measured by centerline curvature was highly comparable with the IVUS-based reconstructions (Table 1 ).

\subsection{Accuracy of 3D OCT vs. 3D IVUS: ESS metrics}

In addition to vessel morphology, we assessed the ability of 3D OCT to provide comparable ESS results with 3D IVUS (Fig. 3; Supplemental Video 1).

Supplementary video related to this article can be found at http://dx.doi.org/10.1016/j.atherosclerosis.2015.04.011.

As shown in Table 1, ESS metrics within each segment were in good agreement across 3D OCT and 3D IVUS with mean differences between methods very close to zero and narrow limits of agreement, as well as high $\mathrm{r}^{2}$ between 0.50 and 0.70 , slopes close to one and intercepts close to zero in linear regression equations. Furthermore, 3D OCT was highly sensitive and specific in identifying subsegments with low ESS $(\leq 0.5 \mathrm{~Pa})$ and high ESS $(>0.5 \mathrm{~Pa})$ using 3D IVUS as gold standard (sensitivity $=0.80$, specificity $=0.80$, positive predictive value $=0.80$, negative predictive value $=0.80$ ).

Collectively, these findings suggest that 3D OCT reconstructions were in high agreement with 3D IVUS in terms of vessel size, shape and ESS calculations.

\subsection{Accuracy of $3 D$ OCT vs. 3D QCA: morphology metrics}

3D OCT reconstructions were further compared to 3D QCA $(\mathrm{n}=7)$, a method that was previously validated and used as reference in the current study (Fig. 3). In global analysis there was a significant agreement between 3D OCT and 3D QCA for total reconstructed artery length and volume (Table 2). Similar to the segmental analysis of 3D OCT vs. 3D IVUS, 3D OCT yielded highly comparable vessel size and shape metrics compared to 3D QCA $(\mathrm{n}=83$, Table 2$)$.

\subsection{Accuracy of 3D OCT vs. 3D QCA: ESS metrics}

With regards to ESS, 3D OCT showed high agreement compared to 3D QCA (Table 2; Fig. 3; Supplemental Video 1). Furthermore, 3D OCT showed high sensitivity and specificity in identifying subsegments with low ESS $(\leq 0.5 \mathrm{~Pa})$ and high ESS ( $>0.5 \mathrm{~Pa})$ compared to 3D QCA (sensitivity $=0.81$, specificity $=0.80$, positive predictive value $=0.81$, negative predictive value $=0.80$ ) .

Collectively, these findings suggest that 3D OCT reconstructions could accurately reproduce the 3D QCA-based measurements of vessel size, shape and ESS. Of note, the agreement between 3D IVUS vs. 3D QCA for global and segmental (morphometric and ESS metrics) analysis was at the same magnitude with the agreement between 3D OCT vs. 3D IVUS and 3D OCT vs. 3D QCA, suggesting that 3D IVUS and 3D QCA were accurate references for the validation of 3D OCT (Supplemental Table 2).

\subsection{Inter- and intra-observer agreement of 3D OCT, $3 D$ IVUS and $3 D Q C A$}

Bland-Altman and linear regression analysis showed that all reconstruction methods had very high inter-observer agreement (Table 3, Supplemental Tables 3 and 4) and intra-observer agreement (Table 4, Supplemental Tables 5 and 6) in terms of artery morphology and ESS parameters $(n=61$ subsegments from 5 arteries). The agreement between 3D OCT vs. 3D IVUS and 3D OCT vs. 3D QCA, was slightly lower, but still comparable, to the inter- and intra-observer agreement of 3D IVUS and 3D QCA, suggesting that 3D OCT can accurately reproduce 3D IVUS or 3D QCA reconstructions.

\subsection{Data processing times}

The data processing time from raw data acquisition to $3 \mathrm{D}$ reconstruction and CFD was approximately three-fold the time of 3D QCA for 3D IVUS and two-fold the time of 3D QCA for 3D OCT (141 \pm 9 vs. $108 \pm 3$ vs. $46 \pm 2 \mathrm{~min}, \mathrm{n}=7, \mathrm{p}<0.01$ for each pair of

Table 1

3D OCT vs. 3D IVUS: Method comparison study using morphology and ESS metrics.

\begin{tabular}{|c|c|c|c|c|c|}
\hline & Bias & Limits of agreement & $\mathrm{r}^{2}$ & $y=\alpha x+\beta$ & $\mathrm{p}$ \\
\hline \multicolumn{6}{|c|}{ Global lumen analysis ( $n=10$ arteries) } \\
\hline Length ( $\mathrm{mm})$ & 0.13 & $-0.59,0.85$ & 0.99 & $\mathrm{y}=1.00 \mathrm{x}+0.04$ & $<0.001$ \\
\hline Volume $\left(\mathrm{mm}^{3}\right)$ & -26.6 & $-113.4,60.17$ & 0.97 & $y=1.28 x-41.61$ & $<0.001$ \\
\hline \multicolumn{6}{|c|}{ Segmental lumen analysis ( $n=114$ subsegments) } \\
\hline \multicolumn{6}{|c|}{ Morphology metrics } \\
\hline Area $\left(\mathrm{mm}^{2}\right)$ & 0.77 & $-2.20,3.72$ & 0.86 & $\mathrm{y}=0.85 \mathrm{x}+1.97$ & $<0.001$ \\
\hline Volume $\left(\mathrm{mm}^{3}\right)$ & 2.31 & $-6.52,11.16$ & 0.86 & $\mathrm{y}=0.85 \mathrm{x}+5.92$ & $<0.001$ \\
\hline Min diameter (mm) & 0.16 & $-0.47,0.80$ & 0.83 & $y=0.75 x+0.86$ & $<0.001$ \\
\hline Max diameter (mm) & 0.13 & $-0.54,0.80$ & 0.82 & $y=0.79 x+0.78$ & $<0.001$ \\
\hline Curvature $\left(\mathrm{mm}^{-1}\right)$ & 0.00 & $-0.07,0.07$ & 0.57 & $y=0.72 x+0.02$ & $<0.001$ \\
\hline \multicolumn{6}{|l|}{ ESS metrics } \\
\hline Mean ESS (Pa) & -0.06 & $-0.67,0.64$ & 0.69 & $y=1.03 x+0.04$ & $<0.001$ \\
\hline Lowest $30^{\circ}$ arc ESS $(\mathrm{Pa})$ & -0.01 & $-0.30,0.28$ & 0.52 & $y=0.90 x+0.07$ & $<0.001$ \\
\hline Highest $30^{\circ}$ arc ESS $(\mathrm{Pa})$ & -0.11 & $-0.87,0.65$ & 0.71 & $y=1.08 x+0.05$ & $<0.001$ \\
\hline
\end{tabular}


Table 2

3D OCT vs. 3D QCA: Method comparison study using morphology and ESS metrics.

\begin{tabular}{|c|c|c|c|c|c|}
\hline & Bias & Limits of agreement & $r^{2}$ & $y=\alpha x+\beta$ & $\mathrm{p}$ \\
\hline \multicolumn{6}{|c|}{ Global lumen analysis ( $n=7$ arteries) } \\
\hline Length $(\mathrm{mm})$ & -0.63 & $-2.80,2.03$ & 0.99 & $\mathrm{y}=1.04 \mathrm{x}-0.76$ & $<0.001$ \\
\hline Volume $\left(\mathrm{mm}^{3}\right)$ & 33.40 & $-17.74,84.51$ & 0.96 & $y=0.89 x-4.90$ & $<0.001$ \\
\hline \multicolumn{6}{|c|}{ Segmental lumen analysis ( $n=83$ subsegments) } \\
\hline \multicolumn{6}{|c|}{ Morphology metrics } \\
\hline Area $\left(\mathrm{mm}^{2}\right)$ & -1.14 & $-3.69,1.42$ & 0.86 & $y=0.64 x+1.46$ & $<0.001$ \\
\hline Volume $\left(\mathrm{mm}^{3}\right)$ & -2.90 & $-9.88,4.08$ & 0.88 & $\mathrm{y}=0.70 \mathrm{x}+3.65$ & $<0.001$ \\
\hline Min diameter (mm) & -0.19 & $-0.78,0.41$ & 0.82 & $\mathrm{y}=0.70 \mathrm{x}+0.64$ & $<0.001$ \\
\hline Max diameter (mm) & -0.34 & $-1.00,0.28$ & 0.81 & $\mathrm{y}=0.67 \mathrm{x}+0.68$ & $<0.001$ \\
\hline Curvature $\left(\mathrm{mm}^{-1}\right)$ & 0.00 & $-0.10,0.10$ & 0.11 & $\mathrm{y}=0.21 \mathrm{x}+0.45$ & $<0.01$ \\
\hline \multicolumn{6}{|l|}{ ESS metrics } \\
\hline Mean ESS (Pa) & 0.02 & $-0.85,0.89$ & 0.62 & $\mathrm{y}=0.88 \mathrm{x}+0.11$ & $<0.001$ \\
\hline Lowest $30^{\circ}$ arc ESS (Pa) & 0.05 & $-0.73,0.82$ & 0.60 & $y=0.95 x+0.07$ & $<0.001$ \\
\hline Highest $30^{\circ}$ arc ESS (Pa) & 0.00 & $-1.16,1.15$ & 0.58 & $\mathrm{y}=0.84 \mathrm{x}+0.17$ & $<0.001$ \\
\hline
\end{tabular}

Table 3

Inter-observer agreement of 3D OCT.

\begin{tabular}{|c|c|c|c|c|c|}
\hline & Bias & Limits of agreement & $r^{2}$ & $\mathrm{y}=\alpha \mathrm{x}+\beta$ & $\mathrm{p}$ \\
\hline \multicolumn{6}{|c|}{ Global lumen analysis ( $n=5$ arteries) } \\
\hline Length $(\mathrm{mm})$ & 0.07 & $-0.48,0.63$ & 0.99 & $\mathrm{y}=0.99 \mathrm{x}+0.46$ & $<0.001$ \\
\hline Volume $\left(\mathrm{mm}^{3}\right)$ & 3.99 & $-21.48,13.49$ & 0.99 & $\mathrm{y}=1.02 \mathrm{x}-10.02$ & $<0.001$ \\
\hline \multicolumn{6}{|c|}{ Segmental lumen analysis ( $n=61$ subsegments) } \\
\hline \multicolumn{6}{|c|}{ Morphology metrics } \\
\hline Area $\left(\mathrm{mm}^{2}\right)$ & 0.19 & $-1.14,0.75$ & 0.98 & $y=1.01 x-0.27$ & $<0.001$ \\
\hline Volume $\left(\mathrm{mm}^{3}\right)$ & 0.44 & $-2.99,2.10$ & 0.99 & $\mathrm{y}=1.01 \mathrm{x}-0.73$ & $<0.001$ \\
\hline Min diameter (mm) & 0.06 & $-0.31,0.19$ & 0.97 & $\mathrm{y}=1.03 \mathrm{x}-0.13$ & $<0.001$ \\
\hline Max diameter (mm) & 0.06 & $-0.31,0.18$ & 0.98 & $\mathrm{y}=1.01 \mathrm{x}-1.06$ & $<0.001$ \\
\hline Curvature $\left(\mathrm{mm}^{-1}\right)$ & 0.00 & $-0.02,0.02$ & 0.85 & $\mathrm{y}=0.93 \mathrm{x}+0.00$ & $<0.001$ \\
\hline \multicolumn{6}{|l|}{ ESS metrics } \\
\hline Mean ESS (Pa) & 0.01 & $-0.24,0.22$ & 0.95 & $y=0.99 x-0.03$ & $<0.001$ \\
\hline Lowest $30^{\circ}$ arc ESS $(\mathrm{Pa})$ & 0.01 & $-0.20,0.19$ & 0.95 & $\mathrm{y}=1.01 \mathrm{x}-0.01$ & $<0.001$ \\
\hline Highest $30^{\circ}$ arc ESS (Pa) & 0.01 & $-0.35,0.37$ & 0.92 & $\mathrm{y}=0.98 \mathrm{x}+0.03$ & $<0.001$ \\
\hline
\end{tabular}

Table 4

Intra-observer agreement of 3D OCT.

\begin{tabular}{|c|c|c|c|c|c|}
\hline & Bias & Limits of agreement & $r^{2}$ & $y=\alpha x+\beta$ & $\mathrm{p}$ \\
\hline \multicolumn{6}{|c|}{ Global lumen analysis ( $n=5$ arteries) } \\
\hline Length $(\mathrm{mm})$ & 0.06 & $-0.70,0.82$ & 0.99 & $\mathrm{y}=0.96 \mathrm{x}+1.46$ & $<0.001$ \\
\hline Volume $\left(\mathrm{mm}^{3}\right)$ & 2.66 & $-4.64,9.97$ & 0.99 & $\mathrm{y}=0.98 \mathrm{x}+7.42$ & $<0.001$ \\
\hline \multicolumn{6}{|c|}{ Segmental lumen analysis ( $n=61$ subsegments) } \\
\hline \multicolumn{6}{|c|}{ Morphology metrics } \\
\hline Area $\left(\mathrm{mm}^{2}\right)$ & 0.03 & $-0.44,0.50$ & 0.99 & $\mathrm{y}=1.00 \mathrm{x}+0.02$ & $<0.001$ \\
\hline Volume $\left(\mathrm{mm}^{3}\right)$ & 0.14 & $-1.15,1.42$ & 0.99 & $\mathrm{y}=1.00 \mathrm{x}+0.16$ & $<0.001$ \\
\hline Min diameter (mm) & 0.01 & $-0.14,0.16$ & 0.99 & $\mathrm{y}=1.00 \mathrm{x}+0.02$ & $<0.001$ \\
\hline Max diameter (mm) & 0.00 & $-0.14,0.13$ & 0.99 & $\mathrm{y}=0.99 \mathrm{x}+0.01$ & $<0.001$ \\
\hline Curvature $\left(\mathrm{mm}^{-1}\right)$ & 0.00 & $-0.03,0.03$ & 0.81 & $\mathrm{y}=0.85 \mathrm{x}+0.01$ & $<0.001$ \\
\hline \multicolumn{6}{|l|}{ ESS metrics } \\
\hline Mean ESS (Pa) & 0.03 & $-0.33,0.28$ & 0.91 & $\mathrm{y}=0.87 \mathrm{x}+0.06$ & $<0.001$ \\
\hline Lowest $30^{\circ}$ arc ESS (Pa) & 0.03 & $-0.43,0.38$ & 0.77 & $y=0.76 x+0.08$ & $<0.001$ \\
\hline Highest $30^{\circ}$ arc ESS (Pa) & 0.01 & $-0.21,0.20$ & 0.97 & $\mathrm{y}=0.96 \mathrm{x}+0.03$ & $<0.001$ \\
\hline
\end{tabular}

comparisons; Table 5). The analysis time with 3D QCA was significantly shorter compared to the other two reconstruction methods given that no invasive imaging was required for 3D QCA. The 3D OCT processing times were significantly shorter compared to $3 \mathrm{D}$ IVUS.

\section{Discussion}

This study presented the methodology of geometrically-correct 3D OCT based on fusion of OCT and biplane angiography and performed an extensive and systematic investigation of the method's accuracy, inter- and intra-observer agreement. Using morphologic and local hemodynamic metrics, 3D OCT was compared head-to- head to 3D IVUS and 3D QCA, which are well-established and validated 3D reconstruction methods. We showed that 3D OCT can accurately, reproducibly and relatively rapidly reconstruct the coronary arteries and calculate ESS. This technique could lay the foundation for combined local hemodynamic (i.e. ESS, FFR) and plaque features assessment in native and stented coronary arteries, potentially creating the perspective for high-risk plaque identification [12].

\subsection{Linear and geometrically-correct 3D OCT}

To date most of the 3D OCT approaches, some of which are commercially available (ILUMIEN PCI Optimization System, St. Jude 
Table 5

Data processing times for each reconstruction method.

\begin{tabular}{|c|c|c|c|c|}
\hline & 3D OCT & 3D IVUS & 3D QCA & $\mathrm{p}$ \\
\hline Raw data acquisition (min) & $9 \pm 1$ & $11 \pm 1$ & $3 \pm 0$ & $<0.001^{\mathrm{a}}$ \\
\hline Cross-sectional image segmentation (min) & $46 \pm 4$ & $77 \pm 9$ & $\mathrm{~N} / \mathrm{A}$ & $<0.01$ \\
\hline Angiogram segmentation (min) & $5 \pm 1$ & $5 \pm 0$ & $1 \pm 0$ & $<0.001^{\mathrm{b}}$ \\
\hline $3 \mathrm{D}$ reconstruction $(\mathrm{min})$ & $6 \pm 0$ & $6 \pm 1$ & $0 \pm 0^{\mathrm{e}}$ & $<0.001^{\mathrm{c}}$ \\
\hline $\mathrm{CFD}(\min )$ & $41 \pm 2$ & $42 \pm 1$ & $42 \pm 2$ & 0.70 \\
\hline Total analysis time (min) & $108 \pm 3$ & $141 \pm 9$ & $46 \pm 2$ & $<0.01^{\mathrm{d}}$ \\
\hline
\end{tabular}

a $p$ refers to 3D QCA vs. 3D OCT and 3D IVUS.

b $\mathrm{p}$ refers to 3D QCA vs. 3D OCT and 3D IVUS.

c $\mathrm{p}$ refers to 3D QCA vs. 3D OCT and 3D IVUS.

d $p$ refers to each pair of comparisons.

ectual time was $0.02 \pm 0.00 \mathrm{~min}$.

Medical, MN, USA), have been based on linear stacking of OCT frames, resulting in non-geometrically correct arterial reconstructions $[25,26]$. Even though those reconstruction approaches, have been fundamental in studying stenting techniques and stent restenosis, they do not allow the assessment of true vessel geometry and local hemodynamics. There have been very few reports on geometrically-correct 3D OCT based on single case studies, none of which validated the reconstruction methodologies $[14,15,27]$. Two other studies utilized geometrically-correct 3D OCT-based CFD to assess FFR [14] and ESS across stent struts [28]. A recent study compared 3D OCT against 3D IVUS and yielded similar findings [29]. However, the current work is the first systematic and detailed investigation of the accuracy and reproducibility of 3D OCT vs. 3D IVUS and 3D OCT vs. 3D QCA in reconstructing coronary arteries and calculating local ESS.

\section{2. $3 D$ OCT vs. $3 D$ IVUS}

We and other groups developed and validated the geometrically-correct 3D IVUS, a technique that was applied in experimental and clinical studies over the last decade allowing the assessment of local ESS, vascular remodeling and plaque burden, thereby advancing our understanding of the role of ESS in the natural history of atherosclerosis $[9,22,23,30]$. In the current study we validated 3D OCT against 3D IVUS. One could argue in theory that 3D OCT is inferior to 3D IVUS given that 3D IVUS is ECG-gated as opposed to 3D OCT. However, our study showed that the (non ECG-gated) 3D OCT was in high agreement with the (ECG-gated) 3D IVUS, suggesting that the absence of ECG gating does not influence significantly the accuracy of 3D OCT reconstructions. This concept is further supported by the elegant study by Fedele et al. who showed satisfactory reproducibility of OCT morphometric measurements stemming from frames acquired during both in systole and diastole, suggesting that no ECG gating is most likely required with such precise and accurate technology [31,32]. In addition to lumen geometry and ESS information, 3D OCT can provide important information about high-risk plaque features (i.e. thin fibrous cap, inflammation, large lipid core), making it a promising technique for high-risk plaque diagnosis (Supplemental Table 7). The data analysis time with 3D OCT was significantly shorter compared to 3D IVUS ( $108 \pm 3$ vs. $141 \pm 9$ min, $\mathrm{p}<0.01$ ) given the superior image quality and subsequently quicker segmentation of OCT vs. IVUS.

\section{3. $3 D$ OCT vs. $3 D Q C A$}

In addition to 3D IVUS, we used 3D QCA to validate 3D OCT. 3D QCA is a technique that can rapidly and accurately reconstruct coronary arteries and can potentially provide local hemodynamic information (i.e. ESS, FFR) [33]. This method has been validated before [10]. The major advantage of 3D QCA is that it does not require invasive imaging (i.e. OCT or IVUS), as it is purely based on two angiographic planes. In the current study, we showed that 3D OCT is comparable to 3D QCA with regard to artery reconstruction and ESS calculations. One could argue that the accuracy of 3D QCA is inferior to 3D IVUS given that 3D QCA is based on two angiographic planes, whereas 3D IVUS is based on accurate tomographic imaging, and therefore 3D QCA cannot be used as reference for the 3D OCT validation. However, the data in the current study do not support this hypothesis. Furthermore, 3D QCA is an appealing 3D reconstruction method given its ability to complete the reconstruction rapidly and almost fully automatically without the need of additional invasive imaging (Supplemental Table 7).

Overall, the current work shows that the agreement of lumen moprhometric and ESS measurements between 3D OCT vs. 3D IVUS and 3D OCT vs. 3D QCA was high and comparable to the inter- and intra-observer agreement of 3D IVUS and 3D QCA, respectively, supporting the high accuracy of 3D OCT. On top of that, even though all three 3D reconstruction modalities had excellent inter- and intra-observer agreement, the 3D OCT exhibited the highest values of agreement, further indicating the robustness of the method.

\subsection{Study limitations}

There were several limitations within the current study. First, we assessed the inter- and intra-observer agreement of the 3D reconstruction modalities, based on repeated reconstructions of the same raw data, and not the inter-study agreement. Had the repeated reconstructions been based on different scans, the potential sources of variation would include time-to-time biological variation, likely decreasing the inter- and intra-observer agreement of the methods. Second, in our study we performed OCT imaging in 35 coronary arteries which were all reconstructed successfully with 3D OCT. Due to technical and cost issues we performed IVUS in a subpopulation of 10 out of 35 arteries, which is an adequate validation cohort. With regard to the 3D QCA, it was performed in 7 out of 35 arteries. The 3D QCA validation software used in the study (QAngio XA 3D RE, Medis Specials bv, Leiden, the Netherlands) works with new generation digital angiography systems, while most of the angiograms $(n=28)$ in our study were acquired with previous generation angiographic systems with no recordings of image acquisition geometrical parameters in DICOM files. Despite the limited number of arteries reconstructed with 3D IVUS or 3D QCA relative to 3D OCT our approach of doing the analyses in multiple subsegments per artery $(\mathrm{n}=114$ for 3D OCT vs. 3D IVUS and $n=83$ for 3D OCT vs. 3D QCA) increased the power of the study substantially. Third, the OCT segmentation was done semiautomatically thus increasing the analysis time. Algorithms that perform automated segmentation of OCT images with limited manual interventions are anticipated to reduce the data processing time, further increasing the feasibility of 3D OCT [12]. This study focused on examining 3-mm-long subsegments, a method which was also used in previous experimental and clinical studies 
$[8,22,23]$, and not on comparing the ESS patterns point-by-point. Therefore, findings from this study should not be directly extrapolated to point-by-point comparisons, where minor errors in coregistration could lead to high differences. The performance of $3 \mathrm{D}$ OCT vs. 3D IVUS/3D QCA across the coronary artery types could not be reliably assessed due to small sample size of the LCX and RCA subgroups. Future studies could address this point. Coronary artery motion could affect the accuracy of 3D OCT. The development of new systems with higher frame acquisition rate and pullback speed could definitely resolve such issues [34]. Finally, 3D OCT could not reconstruct long arterial segments given the inherent limitations of the OCT imaging system used in the current study. However, a typical OCT run of approximately $50 \mathrm{~mm}$ was likely enough to cover the proximal segments of the coronary arteries where the great majority of plaques develop $[35,36]$. Newer OCT imaging systems appear to overcome this limitation by scanning much longer arterial segments.

\subsection{Clinical perspectives}

Geometrically-correct 3D OCT may allow the analysis of pathobiologic plaque characteristics along with the calculation of local hemodynamic microenvironment (i.e. ESS and FFR) [12,14,16]. Studies have shown that low ESS leads to atherogenesis and highrisk plaque development [4]. 3D OCT-based calculation of local ESS and/or FFR in combination with high-risk plaque features, such as thin fibrous cap, inflammation and large lipid pool may facilitate the early identification of high-risk plaque [37]. In addition, geometrically-correct 3D OCT can be used in the investigation of the role of ESS in in-stent restenosis and thrombosis $[28,38]$. Further clinical studies in large cohorts of patients are warranted to investigate those perspectives.

\section{Conclusions}

Geometrically-correct 3D OCT is a new coronary artery imaging modality that can accurately and reproducibly reconstruct the coronary arteries and calculate local ESS. This technique has the potential to allow combined local hemodynamic and plaque morphologic assessement, thereby providing incremental diagnostic and prognostic information for high-risk plaque.

\section{Funding sources}

European Commission, Framework Program 7, Marie Curie International Reintegration Grant, Project: SMILE (grant number: 249303); Behrakis Foundation, Boston, USA; General Secretariat of Research and Technology, Program: Heracleitus II, Athens, Greece; National Institute of Biomedical Imaging and Bioengineering (grant Number K01-EB015868), NIH, Bethesda, USA.

\section{Conflicts of interest and disclosures}

ST had an employment contract with Medis Medical Imaging Systems bv until June 2014. YL is employed by Medis Medical Imaging Systems bv. JHCR is the CEO of Medis Medical Imaging Systems bv and part-time Professor of Medical Imaging at the Leiden University Medical Center, Leiden, the Netherlands.

\section{Appendix A. Supplementary data}

Supplementary data related to this article can be found at http:// dx.doi.org/10.1016/j.atherosclerosis.2015.04.011.

\section{References}

[1] B.E. Claessen, R. Mehran, G.S. Mintz, G. Weisz, M.B. Leon, O. Dogan, J. de Ribamar Costa Jr., G.W. Stone, I. Apostolidou, A. Morales, V. Chantziara, G. Syros, E. Sanidas, K. Xu, J.G. Tijssen, J.P. Henriques, J.J. Piek, J.W. Moses, A. Maehara, G.D. Dangas, Impact of intravascular ultrasound imaging on early and late clinical outcomes following percutaneous coronary intervention with drug-eluting stents, JACC Cardiovasc. Interv. 4 (2011) 974-981.

[2] G.J. Tearney, E. Regar, T. Akasaka, T. Adriaenssens, P. Barlis, H.G. Bezerra, B. Bouma, N. Bruining, J.M. Cho, S. Chowdhary, M.A. Costa, R. de Silva J. Dijkstra, C. Di Mario, D. Dudek, E. Falk, M.D. Feldman, P. Fitzgerald, H.M. Garcia-Garcia, N. Gonzalo, J.F. Granada, G. Guagliumi, N.R. Holm, Y. Honda, F. Ikeno, M. Kawasaki, J. Kochman, L. Koltowski, T. Kubo, T. Kume H. Kyono, C.C. Lam, G. Lamouche, D.P. Lee, M.B. Leon, A. Maehara, O. Manfrini, G.S. Mintz, K. Mizuno, M.A. Morel, S. Nadkarni, H. Okura, H. Otake, A. Pietrasik, F. Prati, L. Raber, M.D. Radu, J. Rieber, M. Riga, A. Rollins, M. Rosenberg, V. Sirbu, P.W. Serruys, K. Shimada, T. Shinke, J. Shite, E. Siegel, S. Sonoda, M. Suter, S. Takarada, A. Tanaka, M. Terashima, T. Thim, S. Uemura, G.J. Ughi, H.M. van Beusekom, A.F. van der Steen, G.A. van Es, G. van Soest, R. Virmani, S. Waxman, N.J. Weissman, G. Weisz, International Working Group for Intravascular Optical Coherence T. Consensus standards for acquisition, measurement, and reporting of intravascular optical coherence tomography studies: a report from the International Working Group for Intravascular Optical Coherence Tomography Standardization and Validation, J. Am. Coll. Cardiol. 59 (2012) 1058-1072.

[3] Y.S. Chatzizisis, A.U. Coskun, M. Jonas, E.R. Edelman, C.L. Feldman, P.H. Stone Role of endothelial shear stress in the natural history of coronary atherosclerosis and vascular remodeling: molecular, cellular, and vascular behavior, J. Am. Coll. Cardiol. 49 (2007) 2379-2393.

[4] J.J. Wentzel, Y.S. Chatzizisis, F.J. Gijsen, G.D. Giannoglou, C.L. Feldman, P.H. Stone, Endothelial shear stress in the evolution of coronary atherosclerotic plaque and vascular remodelling: current understanding and remaining questions, Cardiovasc. Res. 96 (2012) 234-243.

[5] P.H. Stone, A.U. Coskun, S. Kinlay, M.E. Clark, M. Sonka, A. Wahle, O.J. Ilegbusi Y. Yeghiazarians, J.J. Popma, J. Orav, R.E. Kuntz, C.L. Feldman, Effect of endothelial shear stress on the progression of coronary artery disease, vascular remodeling, and in-stent restenosis in humans: in vivo 6-month follow-up study, Circulation 108 (2003) 438-444.

[6] G.D. Giannoglou, Y.S. Chatzizisis, G Sianos, D. Tsikaderis, A. Matakos, V. Koutkias, P. Diamantopoulos, N. Maglaveras, G.E. Parcharidis, G.E. Louridas In-vivo validation of spatially correct three-dimensional reconstruction of human coronary arteries by integrating intravascular ultrasound and biplane angiography, Coron. Artery Dis. 17 (2006) 533-543.

[7] C.J. Slager, J.J. Wentzel, J.C. Schuurbiers, J.A. Oomen, J. Kloet, R. Krams, C. von Birgelen, W.J. van der Giessen, P.W. Serruys, P.J. de Feyter, True 3-dimensional reconstruction of coronary arteries in patients by fusion of angiography and IVUS (ANGUS) and its quantitative validation, Circulation 102 (2000) $511-516$.

[8] P.H. Stone, S. Saito, S. Takahashi, Y. Makita, S. Nakamura, T. Kawasaki A. Takahashi, T. Katsuki, S. Nakamura, A. Namiki, A. Hirohata, T. Matsumura, S. Yamazaki, H. Yokoi, S. Tanaka, S. Otsuji, F. Yoshimachi, J. Honye, D. Harwood, M. Reitman, A.U. Coskun, M.I. Papafaklis, C.L. Feldman, P. Investigators, Prediction of progression of coronary artery disease and clinical outcomes using vascular profiling of endothelial shear stress and arterial plaque characteristics: the PREDICTION Study, Circulation 126 (2012) 172-181.

[9] H. Samady, P. Eshtehardi, M.C. McDaniel, J. Suo, S.S. Dhawan, C. Maynard, L.H. Timmins, A.A. Quyyumi, D.P. Giddens, Coronary artery wall shear stress is associated with progression and transformation of atherosclerotic plaque and arterial remodeling in patients with coronary artery disease, Circulation 124 (2011) 779-788.

[10] S. Tu, Z. Huang, G. Koning, K. Cui, J.H. Reiber, A novel three-dimensional quantitative coronary angiography system: In-vivo comparison with intravascular ultrasound for assessing arterial segment length, Catheter. Cardiovasc. Interv. 76 (2010) 291-298.

[11] L. Goubergrits, E. Wellnhofer, U. Kertzscher, K. Affeld, C. Petz, H.C. Hege Coronary artery WSS profiling using a geometry reconstruction based on biplane angiography, Ann. Biomed. Eng. 37 (2009) 682-691.

[12] Y.S. Chatzizisis, R. Blankstein, P. Libby, Inflammation goes with the flow: implications for non-invasive identification of high-risk plaque, Atherosclerosis 234 (2014) 476-478.

[13] A. Giannopoulos, Y.S. Chatzizisis, G.D. Giannoglou, Optical coherence tomography: an arrow in our quiver, Expert Rev. Cardiovasc. Ther. 10 (2012) 539-541.

[14] C.A. Kousera, S. Nijjer, R. Torii, R. Petraco, S. Sen, N. Foin, A.D. Hughes, D.P. Francis, X.Y. Xu, J.E. Davies, Patient-specific coronary stenoses can be modeled using a combination of OCT and flow velocities to accurately predict hyperemic pressure gradients, IEEE Trans. Biomed. Eng. 61 (2014) 1902-1913.

[15] L.S. Athanasiou, C.V. Bourantas, P.K. Siogkas, A.I. Sakellarios, T.P. Exarchos, K.K. Naka, M.I. Papafaklis, L.K. Michalis, F. Prati, D.I. Fotiadis, 3D reconstruction of coronary arteries using frequency domain optical coherence tomography images and biplane angiography, Conf. Proc. IEEE Eng. Med. Biol. Soc. 2012 (2012) 2647-2650.

[16] S. Tu, S.A. Pyxaras, Y. Li, E. Barbato, J.H. Reiber, W. Wijns, In vivo flow 
simulation at coronary bifurcation reconstructed by fusion of 3-dimensional X-ray angiography and optical coherence tomography, Circ. Cardiovasc. Interv. 6 (2013) e15-e17.

[17] C. Doulaverakis, I. Tsampoulatidis, A.P. Antoniadis, Y.S. Chatzizisis, A. Giannopoulos, I. Kompatsiaris, G.D. Giannoglou, IVUSAngio tool: a publicly available software for fast and accurate 3D reconstruction of coronary arteries, Comput. Biol. Med. 43 (2013) 1793-1803.

[18] C.V. Bourantas, M.I. Papafaklis, L. Athanasiou, F.G. Kalatzis, K.K. Naka, P.K. Siogkas, S. Takahashi, S. Saito, D.I. Fotiadis, C.L. Feldman, P.H. Stone, L.K. Michalis, A new methodology for accurate 3-dimensional coronary artery reconstruction using routine intravascular ultrasound and angiographic data: implications for widespread assessment of endothelial shear stress in humans, EuroIntervention 9 (2013) 582-593.

[19] A. Wahle, P.M. Prause, S.C. DeJong, M. Sonka, Geometrically correct 3-D reconstruction of intravascular ultrasound images by fusion with biplane angiography-methods and validation, IEEE Trans. Med. Imaging 18 (1999) 686-699.

[20] N.S. van Ditzhuijzen, A. Karanasos, N. Bruining, M. van den Heuvel, O. Sorop J. Ligthart, K. Witberg, H.M. Garcia-Garcia, F. Zijlstra, D.J. Duncker, H.M. van Beusekom, E. Regar, The impact of Fourier-Domain optical coherence tomography catheter induced motion artefacts on quantitative measurements of a PLLA-based bioresorbable scaffold, Int. J. Cardiovasc. Imaging 30 (2014) 1013-1026.

[21] Y.S. Chatzizisis, G.D. Giannoglou, A. Matakos, C. Basdekidou, G. Sianos, A. Panagiotou, C. Dimakis, G.E. Parcharidis, G.E. Louridas, In-vivo accuracy of geometrically correct three-dimensional reconstruction of human coronary arteries: is it influenced by certain parameters? Coron. Artery Dis. 17 (2006) $545-551$.

[22] Y.S. Chatzizisis, A.B. Baker, G.K. Sukhova, K.C. Koskinas, M.I. Papafaklis, R. Beigel, M. Jonas, A.U. Coskun, B.V. Stone, C. Maynard, G.P. Shi, P. Libby, C.L. Feldman, E.R. Edelman, P.H. Stone, Augmented expression and activity of extracellular matrix-degrading enzymes in regions of low endothelial shear stress colocalize with coronary atheromata with thin fibrous caps in pigs, Circulation 123 (2011) 621-630.

[23] Y.S. Chatzizisis, M. Jonas, A.U. Coskun, R. Beigel, B.V. Stone, C. Maynard, R.G. Gerrity, W. Daley, C. Rogers, E.R. Edelman, C.L. Feldman, P.H. Stone Prediction of the localization of high-risk coronary atherosclerotic plaques on the basis of low endothelial shear stress: an intravascular ultrasound and histopathology natural history study, Circulation 117 (2008) 993-1002.

[24] J.M. Bland, D.G. Altman, Statistical methods for assessing agreement between two methods of clinical measurement, Lancet 1 (1986) 307-310.

[25] V. Farooq, B.D. Gogas, T. Okamura, J.H. Heo, M. Magro, J. Gomez-Lara, Y. Onuma, M.D. Radu, S. Brugaletta, G. van Bochove, R.J. van Geuns, H.M. Garcia-Garcia, P.W. Serruys, Three-dimensional optical frequency domain imaging in conventional percutaneous coronary intervention: the potential for clinical application, Eur. Heart J. 34 (2013) 875-885.

[26] S. Tu, L. Xu, J. Ligthart, B. Xu, K. Witberg, Z. Sun, G. Koning, J.H. Reiber, E. Regar, In vivo comparison of arterial lumen dimensions assessed by co-registered three-dimensional (3D) quantitative coronary angiography, intravascular ultrasound and optical coherence tomography, Int. J. Cardiovasc. Imaging 28 (2012) 1315-1327.

[27] A.P. Antoniadis, M. Jaguszewski, W. Maier, G.D. Giannoglou, T.F. Luscher, C. Templin, Geometrically correct three-dimensional optical coherence tomography: first self-expanding bifurcation stent evaluation, Eur. Heart J. 34 (2013) 2715 .

[28] C.V. Bourantas, M.I. Papafaklis, L. Lakkas, A. Sakellarios, Y. Onuma, Y.J. Zhang, T. Muramatsu, R. Diletti, P. Bizopoulos, F. Kalatzis, K.K. Naka, D.I. Fotiadis
J. Wang, H.M. Garcia Garcia, T. Kimura, L.K. Michalis, P.W. Serruys, Fusion of optical coherence tomographic and angiographic data for more accurate evaluation of the endothelial shear stress patterns and neointimal distribution after bioresorbable scaffold implantation: comparison with intravascular ultrasound-derived reconstructions, Int. J. Cardiovasc. Imaging 30 (2014) 485-494.

[29] M.I. Papafaklis, C.V. Bourantas, T. Yonetsu, R. Vergallo, A. Kotsia, S. Nakatani, L.S. Lakkas, L.S. Athanasiou, K.K. Naka, D.I. Fotiadis, C.L. Feldman, P.H. Stone, P.W. Serruys, I.K. Jang, L.K. Michalis, Anatomically correct three-dimensional coronary artery reconstruction using frequency domain optical coherence tomographic and angiographic data: head-to-head comparison with intravascular ultrasound for endothelial shear stress assessment in humans, EuroIntervention (2014).

[30] J.J. Wentzel, E. Janssen, J. Vos, J.C. Schuurbiers, R. Krams, P.W. Serruys, P.J. de Feyter, C.J. Slager, Extension of increased atherosclerotic wall thickness into high shear stress regions is associated with loss of compensatory remodeling, Circulation 108 (2003) 17-23.

[31] S. Fedele, G. Biondi-Zoccai, P. Kwiatkowski, L. Di Vito, M. Occhipinti, A. Cremonesi, M. Albertucci, L. Materia, G. Paoletti, F. Prati, Reproducibility of coronary optical coherence tomography for lumen and length measurements in humans (The CLI-VAR [Centro per la Lotta contro l'Infarto-VARiability] study), Am. J. Cardiol. 110 (2012) 1106-1112.

[32] F. Prati, G. Guagliumi, G.S. Mintz, M. Costa, E. Regar, T. Akasaka, P. Barlis, G.J. Tearney, I.K. Jang, E. Arbustini, H.G. Bezerra, Y. Ozaki, N. Bruining, D. Dudek, M. Radu, A. Erglis, P. Motreff, F. Alfonso, K. Toutouzas, N. Gonzalo, C. Tamburino, T. Adriaenssens, F. Pinto, P.W. Serruys, C. Di Mario, Expert review document part 2: methodology, terminology and clinical applications of optical coherence tomography for the assessment of interventional procedures, Eur. Heart J. 33 (2012) 2513-2520.

[33] S. Tu, E. Barbato, Z. Koszegi, J. Yang Z. Sun, N. R. Holm, B. Tar, Y. Li, D. Rusinaru, W. Wijns, J.H. Reiber, Fractional flow reserve calculation from 3-dimensional quantitative coronary angiography and TIMI frame count: a fast computer model to quantify the functional significance of moderately obstructed coronary arteries, JACC Cardiovasc. Interv. 7 (2014) 768-777.

[34] T. Wang, W. Wieser, G. Springeling, R. Beurskens, C.T. Lancee, T. Pfeiffer, A.F. van der Steen, R. Huber, G. van Soest, Intravascular optical coherence tomography imaging at 3200 frames per second, Opt. Lett. 38 (2013) 1715-1717.

[35] P.K. Cheruvu, A.V. Finn, C. Gardner, J. Caplan, J. Goldstein, G.W. Stone, R. Virmani, J.E. Muller, Frequency and distribution of thin-cap fibroatheroma and ruptured plaques in human coronary arteries: a pathologic study, J. Am. Coll. Cardiol. 50 (2007) 940-949.

[36] K. Toutouzas, A. Karanasos, M. Riga, E. Tsiamis, A. Synetos, A. Michelongona, A. Papanikolaou, G. Triantafyllou, C. Tsioufis, C. Stefanadis, Optical coherence tomography assessment of the spatial distribution of culprit ruptured plaques and thin-cap fibroatheromas in acute coronary syndrome, EuroIntervention 8 (2012) 477-485.

[37] R. Vergallo, M.I. Papafaklis, T. Yonetsu, C.V. Bourantas, I. Andreou, Z. Wang, J.G. Fujimoto, I. McNulty, H. Lee, L.M. Biasucci, F. Crea, C.L. Feldman, L.K. Michalis, P.H. Stone, I.K. Jang, Endothelial shear stress and coronary plaque characteristics in humans: combined frequency-domain optical coherence tomography and computational fluid dynamics study, Circ. Cardiovasc. Imaging 7 (2014) 905-911.

[38] B.D. Gogas, S.B. King 3rd, L.H. Timmins, T. Passerini, M. Piccinelli, A. Veneziani, S. Kim, D.S. Molony, D.P. Giddens, P.W. Serruys, H. Samady, Biomechanical assessment of fully bioresorbable devices, JACC Cardiovasc. Interv. 6 (2013) $760-761$. 